\title{
LA RECHERCHE DE L'EAU OXYGÉNÉE DANS LE LAIT
}

\author{
par
}

\author{
JEAN PIEN, J. DÉSIRANT et MHe D. LAFONTAINE
}

Les techniques classiques de recherche de l'eau oxygénée dans le lait reposent sur le principe suivant :

Le lait cru contient des peroxydases qui décomposent les peroxydes ajoutés au lait (eau oxygénée par exemple) en libérant de l'oxygène atomique capable de provoquer certaines oxydations particulières (par exemple : oxydation quinonique de certains polyphénols avec formation de substances colorées).

Il convient de noter qu'un autre mode de décomposition diastasique du perhydrol peut intervenir dans le lait sous l'influence des catalases. Mais, dans ce cas, il y a libération de l'oxygène moléculaire, gazeux, qui ne provoque pas les réactions d'oxydation dont il vient d'être parlé. De sorte que s'il y a, dans le lait, plusieurs mécanismes enzymatiques de décomposition du perhydrol, un seul (celui qui est dû aux peroxydases) est utilisé en vue de la détection de l'eau oxygénée.

Les réactions basées sur ce principe sont assez nombreuses. L'une des plus employées (dérivée de la réaction classique de Dupouy) [1] met en œuvre l'oxydation du gaĩacol qui, en présence du. système peroxyde-peroxydase c'est-à-dire sous l'action de l'oxygène atomique, se transforme en gaïacoquinone et communique au lait une coloration rose saumon plus ou moins intense.

Le mode opératoire le plus courant est le suivant :

A $1 \mathrm{~cm}^{3}$ de lait cru suspect de renfermer de l'eau oxygénée, ajouter $1 \mathrm{~cm}^{3}$ de solution aqueuse de gaïacol saturée à froid. Agiter. En présence d'eau oxygénée il se produit une coloration rose saumon en moins d'une minute à la température ordinaire.

La sensibilité de cette réaction est assez variable suivant l'intensité de l'activité peroxydasique du lait. Elle se situe en moyenne vers $0 \mathrm{~cm}^{3} 4-0 \mathrm{~cm}^{3} 6$ parfois $1 \mathrm{~cm}^{3}$ d'eau oxygénée à 12 volumes par litre de lait c'est-à-dire 15 à 20 , voire 35 parties de peroxyde d'hydrogène par million.

$$
* * *
$$

\section{Critique des méthodes classiques basées sur l'intervention des peroxydases}

On peut faire à ces techniques trois objections :

$1^{\circ}$ D'abord, elles ne sont pas applicables au cas du lait pasteurisé où le chauffage a détruit les peroxydases et où, par consé- 
quent, en présence d'eau oxygénée, la réaction précédente n'a pas lieu. Rappelons que c'est précisément là le principe de certaines méthodes de contrôle de la pasteurisation : on ajoute à un petit volume de lait, de l'eau oxygénée et l'un des réactifs destinés à donner une coloration par oxydation; si la réaction est positive (par suite de la présence de peroxydase) on conclut que l'on est en présence de lait cru ou d'un mélange de lait pasteurisé et de lait cru; si la réaction est négative en conlut que l'on est en présence de lait pasteurisé ou, du moins, de lait dans lequel la peroxydase a été détruite. (Réaction de Dupouy proprement dite, de Storch, de Rothenfusser, de Schern-Schellhaze, etc.) $[2,3,4]$.

Ces réactions sont donc inapplicables au dépistage de l'eau oxygénée dans le lait pasteurisé. On a depuis longtemps tourné cette difficulté en ajoutant un peu de peroxydase au lait pasteurisé. Dans ce cas, en effet, la réaction redevient positive en présence de perhydrol, comme s'il s'agissait de lait cru. Comme source de peroxydase "extérieure" on fait appel soit à un léger apport de lait cru (à condition d'avoir vérifié au préalable qu'il ne renfermait pas d'eau oxygénée) soit de salive comme le suggère TAPERNoux [5], soit de macérations de certains végétaux, purifiées ou non : poire, pomme de terre recommandée par Fouassier [6], oignon dont nous avons reconnu qu'il pouvait convenir à condition de le choisir, car certaines variétés ne renferment paś de peroxydase, ou très peu. Mais ces apports de peroxydase présentent plusieurs inconvénients :

Les laboratoires n'ont pas toujours sous la main du lait cru frais.

La salive présente des teneurs très variables en peroxydase. Parfois elle n'en renferme pas, ainsi que le note TApervoux luimême [5].

L'extraction des peroxydases végétales est une complication et parfois une difficulté : Si l'on s'en tient à la préparation extemporanée de macérations, on obtient des solutions de peroxydases de titre très variable, se conservant très mal; si l'on veut séparer la diastase à l'état de pureté en vue de sa conservation, on se trouve placé devant de nouveaux problèmes.

Dans tous les cas, donc, il y a des difficultés, voire des incertitudes. C'est là un premier inconvénient de ces méthodes.

$2^{\circ}$ En second lieu il arrive que certains laits orus additionnés de quantités importantes d'eau oxygénée ne renferment plus de peroxydase après un temps assez court. Nous avons en effet constaté que le perhydrol à haute dose (par exemple 4 ou $5 \mathrm{~cm}^{3}$ d'eau oxygénée à 120 volumes par litre de lait) détruisait la peroxydase 
en un ou deux jours dans le lait cru. Les choses se passent alors comme si l'on avait affaire à du lait pasteurisé. On pourrait, certes, comme dans le cas précédent, détecter la présence éventuelle d'eau oxygénée par un apport de peroxydase extérieure; mais dans l'ignorance où l'on se trouve de la quantité approximative d'eau oxygénée présente, on ne songera pas nécessairement à effectuer cette addition de peroxydase et en présence de la réaction négative on conclura à l'absence d'eau oxygénée... alors qu'il y en a beaucoup. Par ailleurs, dans ce cas également, la réaction de Dupouy proprement dite sera faussée: dans ce lait cru elle sera négative comme dans le lait pasteurisé ; si l'on n'y prend pas garde on risquera, là également, d'aboutir à une conolusion fausse. (C'est pourquoi, en présence d'un résultat négatif nous conseillons, dans le cas de l'emploi de ces réactions, de refaire l'essai après addition de peroxydase extérieure : qu'il s'agisse de lait cru "hyper-oxygéné " ou de lait pasteurisé, la présence de perhydrol conduira alors à une réaction positive). Quoi qu'il en soit, cet ensemble de faits est parfois déroutant surtout si l'on a affaire, comme nous le montrerons plus tard, à des produits laitiers riches en matière grasse et acides où la peroxydase, sans être détruite, est inactivée.

$3^{\circ}$ Enfin ces techniques ne sont ni très fjdèles ni très sensibles. En laissant de côté le fait - sur lequel nous reviendrons ci-après de la rapidité de destruction de l'eau oxygénée par la catalase du lait oru et pour s'en tenir à la recherche instantanée du perhydrol que l'on vient d'ajouter au lait, on constate, comme nous l'avons indiqué plus haut, que la réaction au gaĩacol ne permet de déceler dans la majorité des cas que 15 à 20 parties de $\mathrm{H}^{2} \mathrm{O}^{2}$ par million dans le lait.

Ce défaut de sensibilité vis-à-vis des peroxydes a déjà été reconnu par EYRARD et JouFFret [7] qui recommandent l'emploi de la réaction de Schern-Sehellhaze modifiée d'une façon heureuse par ces auteurs. Néanmoins cette réaction n'échappe pas aux critiques précédentes puisqu'elle est basée, elle aussi, sur l'intervention des peroxydases.

Pour toutęs ces raisons, nous pensons que les méthodes classiques de recherche de l'eau oxygénée dans le lait sont loin d'être parfaites et qu'il y aurait intérêt à les remplacer par une technique ne faisant pas appel aux actions enzymatiques.

$$
*^{*} *
$$

Mise au point d'un test qualitatif basé sur l'oxydation

Il vient immédiatement à l'esprit de chercher à utiliser pour la 
recherche (voire le dosage) de l'eau oxygénée dans le lait, l'une des méthodes purement chimiques utilisées pour le titrage du perhy. drol dans ses solutions aqueuses.

Ces méthodes sont nombreuses : titrage classique au permanganate, utilisation du mélange sulfate ferreux + sulfocyanure (Horst [8]), de la coloration jaune obtenue avec le molybdate d'ammonium en milieu acide (IsAACS [9]), formation du sel ferrique de l'acide dioxytartrique (réaction de Fenton, reprise par Denigès [10]), etc. Ces méthodes ne conviennent pas ou conviennent mal à la recherche de l'eau oxygénée dans le lait.

En revanche, l'oxydation de l'iodure de potassium, avec mise en évidence ou titrage de l'iode libéré, paraît devoir fournir une excellente solution échappant entièrement aux critiques formulées plus haut.

L'idée, d'ailleurs, n'est pas nouvelle : déjà en 1927, C. D. How aRd et N. Civen [11] préconisaient cette méthode qu'ils considéraient comme la meilleure et dont ils disaient qu'elle était "entièrement digne de confiance "... Plus récemment les Italiens L. Morandi et L. Satta [12] ont, sur eette même base, donné une méthode de dosage de l'eau oxygénée dans le lait dont, à titre documentaire, nous rappelons brièvement le mode opératoire :

\section{Dosage de l'eau oxygénée dans le lait (Morandi et Satta)}

Dans une fiole non jaugée de $200 \mathrm{~cm}^{3}$, introduire $100 \mathrm{~cm}^{3}$ de lait. Porter à $40^{\circ} \mathrm{C}$. Ajouter $10 \mathrm{~cm}^{3}$ d'acide chlorhydrique dilué au quart pour coaguler la caséine. Refroidir. Filtrer. Prélever $55 \mathrm{~cm}^{3} \mathrm{du}$ filtrat limpide et les introduire dans une fiole d'un litre bouchant à l'émeri. Ajouter $5 \mathrm{~cm}^{3}$ d'acide chlorhydrique concentré et 1 gramme d'iodure de potassium eristallisé. Agiter. Laisser en contact 30 à 40 minutes en agitant fréquemment. Ajouter $500 \mathrm{~cm}^{3}$ d'eau. Titrer par l'hyposulfite N/10 en présence d'empois d'amidon.

$1 \mathrm{~cm}^{3} \mathrm{~d}$ 'hyposulfite $\mathrm{N} / 10$ correspond à $1 \mathrm{mgr}$. 7 de peroxyde d'hydrogène $\left(\mathrm{H}^{2} \mathrm{O}^{2}\right)$.

Remarque. - Ajoutons que si l'on veut traduire le poids $\mathrm{P}$ de peroxyde d'hydrogène ainsi dosé en centimètres cubes d'eau oxygénée à $T$ volumes (1), il faut procéder au calcul suivant : puisque 34 grammes (une molécule) de $\mathrm{H}^{2} \mathrm{O}^{2}$ libèrent un atome d'oxygène c'est-à-dire un demi-v́olume moléculaire ou 11 l. 2 d'oxygène, le volume d'oxygène moléculaire en puissance dans $\mathrm{P}$ grammes de $\mathrm{H}^{2} \mathrm{O}^{2}$ vaut :

$$
\frac{11,2 \times \mathrm{P}}{34} \text { litres de } \mathrm{O}^{2}
$$

(1) Rappelons que le titre d'une eau oxygénée exprime le volume d'oxygène moléculaire susceptible d'être dégagé par la dissociation complète de l'unité de volume de l'eau oxygénée. 
Le volume d'eau oxygénée de titre T correspondant s'exprime done par :

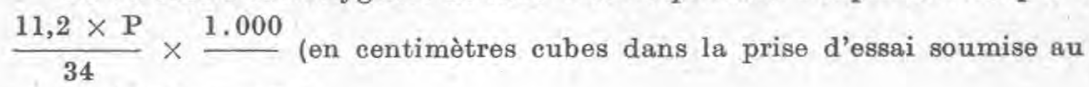
titrage à l'hyposulfite)

C'est par un calcul analogue que l'on peut connaître la richesse en $\mathrm{H}^{2} \mathrm{O}^{2}$ des eaux oxygénées commerciales de divers titres (1).

Mais nous ne cherchons pas ici une méthode de dosage rigoureuse, inutile pour le but poursuivi. Nous désirons seulement aboutir à un test qualitatif rapide, simple et fidèle permettant de déceler instantanément (voire en dehors du laboratoire) la présence de traces de l'eau oxygénée dans le lait cru ou pasteurisé.

Nous avons adopté le principe de l'oxydation de l'iodure de potassium et avons finalement abouti à la technique très simple que voici :

\section{Test qualitatif proposé pour la recherche de l'eau oxygénée dans un lait quelconque}

10 Dans un tube à essai introduire successivement:

$2 \mathrm{em}^{3}$ de lait (cru ou pasteurisé, neutre ou acide);

$2 \mathrm{~cm}^{3}$ d'acide chlorhydrique à $1 \%$. Agiter ;

$2 \mathrm{~cm}^{3}$ d'iodure de potassium à $10 \%$.

$2^{\circ}$ Porter le contenu du tube à $80-90^{\circ}$ par immersion au bainmarie bouillant pendant une minute. Refroidir rapidement et eomplètement sous courant d'eau froide.

$3^{\circ}$ Ajouter $2 \mathrm{~cm}^{3}$ d'empois d'amidon à $1 \%$.

En présence d'eau oxygénée il se produit immédiatement une coloration bleue dont l'intensité est proportionnelle à la quantité de perhydrol présente dans le lait examiné.

Sensibilité de la réaction. Cette réaction permet de déceler à la limite de sensibilité : $0 \mathrm{~cm}^{3} 1-0 \mathrm{~cm}^{3} 2$ d'eau oxygénée à 12 volumes dans un litre de lait (ou $0 \mathrm{~cm}^{3} 1-0 \mathrm{~cm}^{3} 2$ de perhydrol à 120 volumes). Cette limite correspond à $3-6 \mathrm{mgr}$. de peroxyde d'hydrogène $\left(\mathrm{H}^{2} \mathrm{O}^{2}\right)$ par litre soit : 3 à 6 p. p. m.

\section{Remarques :}

10 Le chauffage du mélange lait $+\mathrm{HCl}+\mathrm{KI}$ a pour résultat de provoquer l'oxydation immédiate de l'iodure de potassium (qui, à froid, demanderait 30 à 40 minutes).

(1) Ce calcul donne:

Pour l'eau oxygénée à 10 volumes: $3 \mathrm{gr} .03 \mathrm{de} \mathrm{H}^{2} \mathrm{O}^{2} \mathrm{dans} 100 \mathrm{~cm}^{3} \mathrm{~d}$ 'eau oxygénée ; Pour l'eau oxygénée à 12 volumes : $3 \mathrm{gr} .64 \mathrm{de} \mathrm{H}^{2} \mathrm{O}^{2} \mathrm{dans} 100 \mathrm{~cm}^{3} \mathrm{~d}$ 'eau oxygénée ; Pour l'eau oxygénée à 110 volumes : $33 \mathrm{gr} .4$ de $\mathrm{H}^{2} \mathrm{O}^{2}$ dans $100 \mathrm{~cm}^{3}$ d'eau oxygénée ; Pour l'eau oxygénée à 130 volumes : $39 \mathrm{gr} .4$ de $\mathrm{H}^{2} \mathrm{O}^{2}$ dans $100 \mathrm{~cm}^{3}$ d'eau oxygénée. 
2o Il n'y a pas intérêt à vtiliser des volumes plus faibles de réactifs plus concentrés (dans le but d'augmenter la sensibilité de la réaction). L'expérience a montré que les conditions indiquées cidessus correspondent à l'optimum.

$3^{\circ}$ La réaction vaut pour les laits pasteurisés comme pour les laits crus attendu qu'il n'est pas fait appel à une intervention diastasique.

$4^{\circ}$ La réaction est généralement valable quelle que soit l'acidité du lait. Elle est d'ailleurs généralement négative dans les laits acides ayant reçu de l'eau oxygénée à l'état frais, pour la simple raison que l'acidification se produit après la disparition spontanée du perhydrol. Quoi qu'il en soit, si de l'eau oxygénée était ajoutée à un lait en cours d'acidification, l'acidité acquise ne gênerait pas la mise en évidence de l'eau oxygénée.

$5^{0}$ La réaction est inapplicable dans les laits bichromatés où les réactions sont positives, même en l'absence de perhydrol par suite de l'oxydation de l'iodure de potassium par le bichromate (en milieu acide).

$6^{\circ}$ Le formol rend également impossible la recherche de l'eau oxygénée dans les laits.

\section{Distinction de l'eau oxygénée et des hypochlorites dans le lait}

La réaction précédente n'est évidemment pas spécifique du perhydrol. De nombreux oxydants peuvent libérer l'iode de l'iodure de potassium ; c'est le cas notamment du chlore et des hypochlorites dont cette même réaction constitue un excellent moyen de dépistage.

Il y avait donc là une objection à l'emploi de la méthode. L'objection vaut d'ailleurs identiquement pour les réactions basées sur l'oxydation des polyphénols (gaïacol par exemple) en présence ou en l'absence de peroxydases : le gaïacol donne une coloration rouge dans les laits additionnés d'hypochlorites.

En ce qui concerne la réaction à l'iodure de potassium, cette difficulté peut heureusement être tournée. En effet :

Nous avons constaté que si l'on provoque la coagulation par un acide de laits renfermant soit de l'eau oxygénée, soit de l'eau de javel et si l'on sépare par filtration le sérum du coagulum, on constate que l'eau oxygénée passe dans le sérum alors que le chlore est fixé par la caséine et est absent dans le sérum.

Si donc on veut faire la distinction entre ces deux substances, on procédera de la façon suivante : 
10 Exécuter le test qualitatif dans les conditions indiquées plus haut. Si le résultat est négatif : il n'y a ni eau oxygénée, ni hypochlorites (ou il y a seulement des quantités inférieures à la limite de sensibilité des réactions) (1). Si le résultat est positif, le lait contient l'un ou l'autre des deux antiseptiques.

$2^{\circ}$ Dans ce dernier cas répéter le même test en ayant soin de filtrer le mélange après l'apport d'acide chlorhydrique. Dans le filtrat ajouter l'iodure de potassium, faire bouillir, refroidir, ajouter l'empois. Si la réaction est positive, on avait affaire à de l'eau oxygénée. Si elle est négative, on avait affaire à un hypochlorite.

Nota. - Le chlore est rapidement complexé par la caséine et indécalable par cette réaction en moins d'une heure. Donc le problème de la distinction des deux antiseptiques par la réaction à l'iodure de potassium ne se pose pas dans la pratique courante.

$$
*^{*} *
$$

\section{Utilisation d'un papier réactif pour la détection de l'eau oxygénée et des hypochlorites}

Etant donné l'extrême rapidité avec laquelle l'eau oxygénée disparaît dans les laits crus (voir ci-après) la recherche éventuelle doit en être faite le plus près possible de l'heure de la fraude. Le délai de transport d'un échantillon au laboratoire peut être suffisant dans bien des cas pour que de petites quantités d'eau oxygénée aient entièrement disparu.

Il peut donc être utile, dans certains cas, soit d'effectuer la réaction sur place (à l'aide d'une trousse permettant d'exécuter la réaction précédente) soit, plus simplement encore, de plonger dans le lait suspect une bande de papier iodo-amidonné.

Malheureusement cette technique n'est pas très sensible, car l'oxydation de l'iodure de potassium par l'eau oxygénée ne se produit bien qu'en milieu acide. Nous avons donc songé à créer un papier imprégné d'iodure de potassium, d'empois d'amidon et d'un acide faible non hygroscopique (acide oxalique, acide borique, acide salicylique...). L'expérience a montré qu'un tel papier se conservait mal : il s'y produit assez rapidement une libération d'iode qui le colore.

Nous avons tourné la difficulté en utilisant deux papiers : l'un imprégné d'iodure et d'empois (papier iodo-amidonné classique),

(1) Sensibilité de la réaction dans le cas des hypochlorites : $2 \mathrm{em}^{3}$ d'eau de javel à $12^{\circ}$ chlorométriques paı litre de lait (immédiatement après l'addition). Après 30 minutes on ne décède pratiquement pas $5 \mathrm{~cm}^{3}$ d'eau de javel par litre de lait. 
l'autre imprégné d'une solution d'acide oxalique à $10 \%$, séché à l'air libre.

Mode d'emploi.

Immerger le papier iodo-amidonné dans le lait à examiner.

L'appliquer ensuite contre le papier "acide». Les maintenir en contact pendant 30 minutes. L'apparition d'une coloration bleue violacée révèle la présence dans le lait d'eau oxygénée.

Sensibilité : $0 \mathrm{~cm}^{3} 5 . \mathrm{H}^{2} \mathrm{O}^{2}$ à 12 volumes par litre de lait.

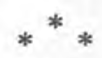

\section{Application du test proposé à l'étude de la vitesse de dissociation de l'eau oxygénée dans le lait}

Il est bien connu que l'eau oxygénée ajoutée au lait s'y détruit rapidement. Les conditions de cette disparition sont importantes à connaître pour le dépistage des fraudes. Or les avis des auteurs sont divergents sur ce point. Nous avons cru utile de revenir sur cette question.

Tout d'abord une distinction fondamentale s'impose suivant qu'il s'agit de lait cru ou de lait pasteurisé.

Dans le lait cru, en effet, il y a au moins trois causes de dissociation du perhydrol :

1. Les catalases naturelles (d'origine leucocytaire) qui décomposent le perhydrol en libérant de l'oxygène moléculaire.

2. Les peroxydases naturelles (libres ou d'origine leucocytaire ou cellulaire) qui décomposent le perhydrol en libérant de l'oxygène atomique.

3. Les microorganismes dont certains secrètent des catalases, des peroxydases, des réductases, etc.

Dans le lait pasteurisé, en revanche, toutes les diastases sont en principe détruites, à condition que la température et la durée de pasteurisation aient été suffisantes : les peroxydases contenues dans les leucocytes sont plus difficiles à détrüire que la peroxydase libre. Un ehauffage insuffisamment prolongé peut donner immédiatement une réaction de Storch ou de Dupouy négative, mais celle-ci peut redevenir positive ultérieurement par suite de la sortie progressive des diastases des corps cellulaires (ORLA-JENSEN et WiNTHER [13]). Pour d'autres auteurs cette "régénération" aurait une origine toute différente (SchULTz et SyDow [14]).

Quoi qu'il en soit, l'eau oxygénée est, pour cette raison, beaucoup plus stable dans le lait pasteurisé que dans le lait cru. Néanmoins on constate des variations considérables dans la durée de cette stabilité. Cela tient, selon nous, à deux causes : 
1. Les germes sporulés non détruits par la pasteurisation peuvent sécréter des catalases. Fouassier [6] a montré que les subtilis, les tyrothrix manifestaient un pouvoir catalasique élevé. L'importance de ce facteur est fonction des conditions plus ou moins hygiéniques qui ont présidé à la récolte du lait.

2. Les germes réintroduits dans le lait pasteurisé à la faveur des contaminations apportent à leur tour de nouvelles sources d'activité catalasique et peroxydasique.

Les laits pasteurisés les plus propres, bactériologiquement, seront done ceux où l'eau oxygénée se díssociera le plus lentement. L'expériencé a confirmé cette manière de voir : si, en effet, la pasteurisation est faite au laboratoire dans de bonnes conditions d'asepsie, la stabilité du perhydrol augmente notablement.

En dehors de cette question de chauffage et de recontamination, la notion de la durée de dissociation du perhydrol (exprimée par le temps au bout duquel les réactions deviennent négatives) est influencée par la quantité d'eau oxygénée introduite dans le lait et par la température.

Tous nos essais ont été effectués à $18^{\circ}$. Nous avons introduit dans des laits orus et dans des laits pasteurisés des doses croissantes d'eau oxygénée à 12 volumes exactement, couvrant et dépassant les doses probables de la fraude et nous avons suivi les réactions dans le temps jusqu'à disparition totale du perhydrol.

\section{Résultats obtenus dans le cas du lait cru.}

Les auteurs qui se sont occupés de cette question donnent, pour des doses d'eau oxygénée à 12 volumes variant de 1 à $15 \mathrm{~cm}^{3}$ par litre de lait cru, des durées de dissociation allant de 3 heures à 7 heures à la température ordinaire $[5,7,12]$.

Voici quelques-uns des résultats que nous avons obtenus : voir tableau ci-après.

\section{Commentaires :}

$1^{0}$ Les très faibles doses d'eau oxygénée disparaissent très rapidement (en moins d'une heure ou en quelques heures).

$2^{\circ}$ Les doses susceptibles d'être employées par les fraudeurs (par exemple de 1 à $10 \mathrm{~cm}^{3}$ d'eau oxygénée à 12 volumes par litre de lait) disparaissent, suivant les laits, en moins d'un jour ou en moins de deux jours.

$3^{0}$ Les doses fortes (10 à $30 \mathrm{~cm}^{3}$ par litre correspondant à $1-3 \mathrm{~cm}^{3}$ de perhydrol à 120 volumes par litre de lait) peuvent encore être décelées pendant plusieurs jours.

$4^{\circ}$ On constate une très grande irrégularité dans la vitesse de 
TEMPS AU BOUT DUQUEL LES RÉACTIONS ONT CESSÉ D'ÊTRE POSITIVES (A 18)

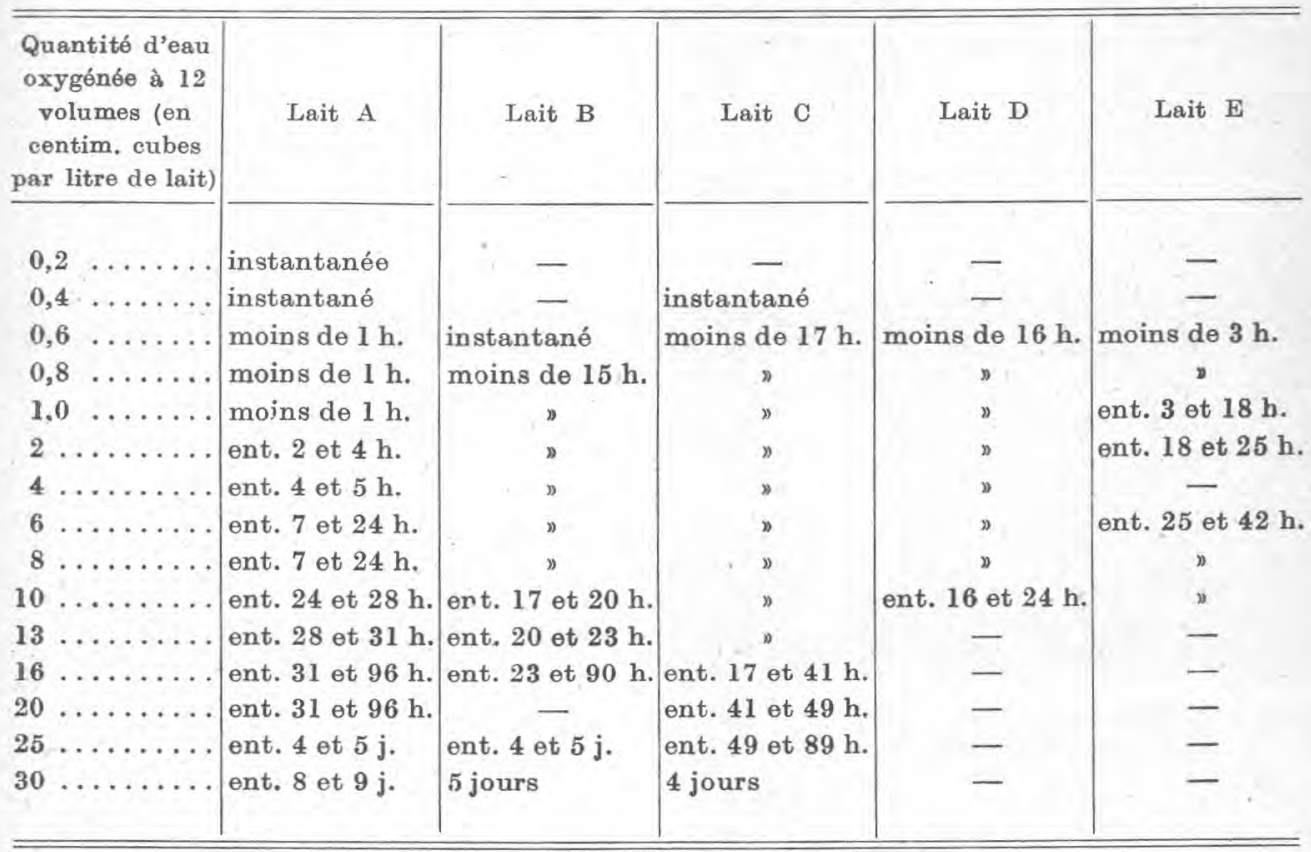

dissociation de l'eau oxygénée d'un lait cru à l'autre. Cela tient évidemment aux différences dans la nature et la quantité de la charge microbienne.

$5^{0}$ Les autres auteurs ont constaté des durées de dissociation beaucoup plus brèves parce qu'ils utilisaient une réaction moins sensible. La réaction à l'iodure de potassium permet de retrouver de plus faibles quantités d'eau oxygénée que les réactions classiques et pendant les temps plus longs.

\section{Résultats obtenus dans le cas des laits pasteurisés.}

Les rares auteurs qui se sont penchés sur ce problème donnent, pour des teneurs en eau oxygénée à 10-12 volumes de 2 à $4 \mathrm{~cm}^{3}$ par litre de lait, des durées de dissociation comprises entre 10 et 30 heures.

Nos résultats, obtenus sur une plus vaste échelle et avec une méthode de détection plus sensible, sont très différents. En voici quelques-uns à titre d'exemple (laits pasteurisés de bonne qualité bactériologique) : 


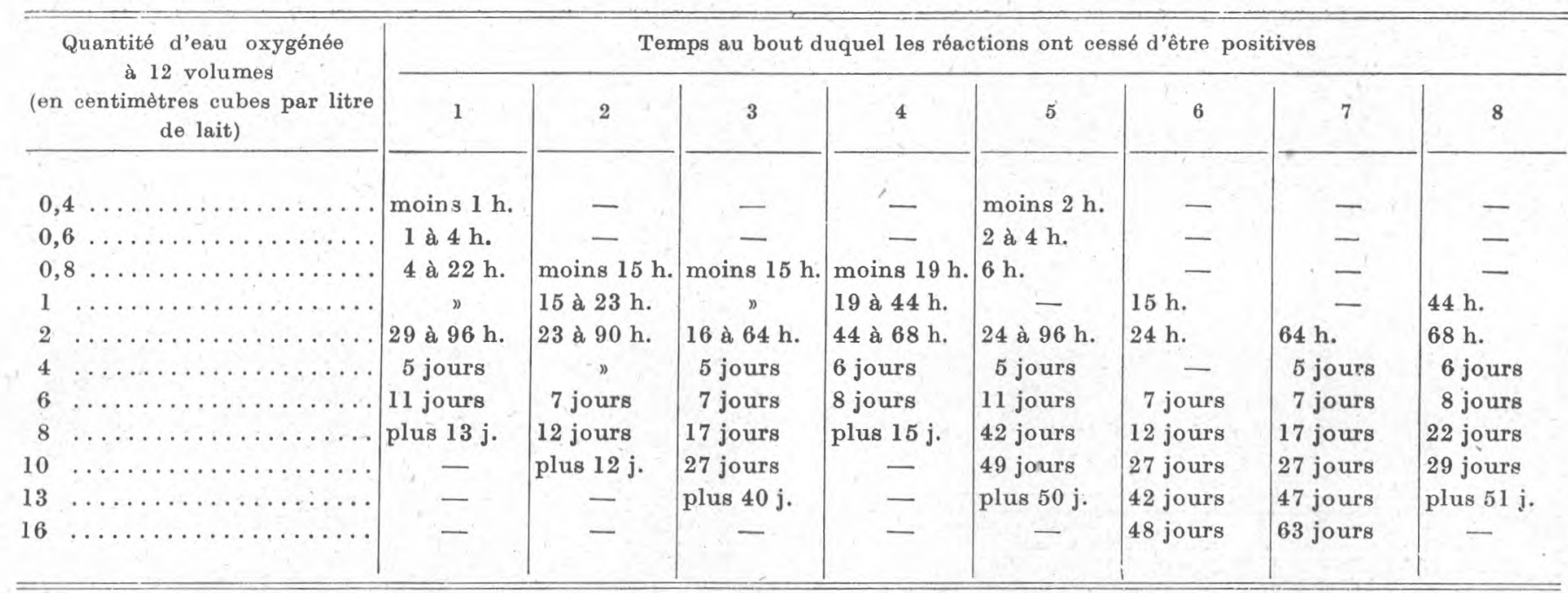




\section{Commentaires :}

$1^{\circ}$ Les faibles doses d'eau oxygénée disparaissent en moins de 24 heures.

$2^{\circ}$ Les doses susceptibles d'être utilisées par les fraudeurs disparaissent en des temps qui varient entre 1 et 30 jours. Par exemple : $2 \mathrm{~cm}^{3}$ se retrouvent pendant 2 à 4 jours ; $6 \mathrm{~cm}^{3}$ pendant 5 à 10 jours, $10 \mathrm{~cm}^{3}$ donnent encore une réaction positive après un mois et même davantage.

$3^{\circ}$ Des quantités plus importantes peuvent parfois être détectées après 50 et 60 jours dans les laits pasteurisés de bonne qualité.

$4^{\circ}$ On note encore ici une assez grande variabilité dans les résultats due aux différences de composition bactériologique des laits (nature et quantité de la flore résiduelle ou réintroduite à la faveur des contaminations). Des laits pasteurisés très contaminés donnent des résultats moins favorables.

$5^{\circ}$ Quoi qu'il en soit, les durées de dissociation que nous avons pu observer en utilisant la réaction à l'iodure de potassium sont beaucoup plus longues que celles qui ont été constatées par d'autres auteurs. Ce résultat est de nature à faciliter le dépistage de la fraude.

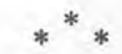

\section{Résumé et conclusions pratiques}

10 Les méthodes classiques de recherche de l'eau oxygénée dans le lait présentent des inconvénients qui ont été exposés en détail.

$2^{\circ} \mathrm{A}$ ces méthodes nous proposons de substituer l'emploi d'un test plus sensible et d'exécution plus facile basé sur l'oxydation de l'iodure de potassium par le perhydrol avec mise en évidence de l'iode libéré. Ce test peut être appliqué en dehors du laboratoire en utilisant deux papiers réactifs dont la préparation est décrite.

$3^{\circ}$ Cette technique convient à la recherche de l'eau oxygénée dans les laits crus et dans les laits pasteurisés sans modification.

$4^{\circ}$ La dissociation de l'eau oxygénée dans le lait sous l'influence des catalases et des peroxydases cellulaires ou microbiennes rendait difficile la recherche de cet antiseptique, notamment dans le lait cru. L'emploi de cette réaction plus sensible permet de mettre en évidence la présence de petites quantités d'eau oxygénée dans le lait cru pendant un ou deux jours (suivant l'état de contamination du lait). Dans les laits pasteurisés cette réaction donne des résultats positifs pendant plusieurs jours (ou même plusieurs semaines) 
suivant les quantités d'eau oxygénée présentes et suivant le degré de recontamination du lait.

$5^{\circ}$ Le délai pendant lequel la recherche de la fraude est possiblese trouve donc augmenté et la possibilité d'effectuer la réaction en dehors du laboratoire (à l'aide des papiers réactifs) permettra un dépistage encore plus précoce donc plus sensible et plus sûr.

\title{
BIBLIOGR APHIE
}

[1] Réaction de Dupouy. Gazette des Sciences médicales de Bordeaux, 1902, p. 566.

[2] 40e Rapport du Laboratoire d'essais agricoles du Danemark, 1898.

[3] Méthode de Rothenfusser. Die praktische Milchprüffung, K. Schneider, p. 58 (Bern, 1938).

[4] Schern et Schellhaze. Berliner Tiérärz. Wochenschr., 1911, p. 868.

[5] TAPERnoux. Le Lait, 1928, p. 410.

[6] Fouassier. C.R.A.S., t. CLXX, no 2, p. 145.

[7] EYrard et Jouffret. Le Lait, 1943, p. 141.

[8] F. HoRst. Bull. Soc. Chimique, 1922, no 1, p. 193.

[9] IsaAcs. Journal American Chem. Soc., 1922, p. 1662.

[10] Denigìs. Ann. Che. anal., 1917, p. 193.

[11] C. D. How ARD et N. Civen. Ind. and Engin. Chem., 19, 1927, p. 161.

[12] L. Morandi et L. Satta. Médicina e Biologia., III, 1943, 21.

[13] Orla-Jensen et Winther. Le Lait, 1935, p. 247.

[14] M. E. Schultz et G. Sydow. Kieler Milchwirtschaft. Forseh-Berichte, 3, 6. 673, 1951.

\section{IDENTIFICATION DES BACTÉRIES LACTIQUES, SELON ORLA-JENSEN}

\author{
par

\section{ERnEsto GUNTHER KASDORF}

Cet exposé, résultant d'un très important travail de compilation (1) $a$ été publié (2) par E. GÜNTHER KASDORF pour mettre à la portée des techniciens de langue espagnole l'ouvre de l'éminent savant danois S. OrLA-Jensen "The lactic acid bacteria, Copenhague, 1919 ; Les vraies bactéries lactiques - volume complémentaire Copenhague 1943 ".

Les propriétés prises comme caractéristiques pour la description

(1) L'Auteur rend hommage à la coopération accordée par M. l'Ingénieur agronome Santos-Soriano, Professeur titulaire de l' l'Institut de Microbiologie Agricole de la Faculté agronomique vétérinaire de Buenos-Aires, ainsi qu'à la collaboration de M. l'Ingénieur agronome I. Benchetrit, Chef des travaux pratiques et de M. l'Ingénieur J. L. Mulvany, Professeur titulaire de la Chaire de Laiterie à la Faculté agronomique de La Plata.

(2) La Industria Letchera, $\mathrm{n}^{\circ} 394$, p. 231. 